\title{
Maintenance scheduling for multi-component systems with hidden failures
}

\author{
Bin Liu $^{\text {a,* }}{ }^{\text {, Ruey-Huei Yeh }}{ }^{\text {b }}$, Min Xie ${ }^{\text {a,c }}$, Fellow, IEEE, Way Kuo ${ }^{\text {a }}$, Fellow, IEEE \\ ${ }^{a}$ Department of Systems Engineering and Engineering Management, \\ City University of Hong Kong, Kowloon, Hong Kong \\ ${ }^{\mathrm{b}}$ Department of Industrial Management, \\ National Taiwan University of Science and Technology, Taipei, Taiwan \\ ${ }^{\mathrm{c}}$ Shenzhen Research Institute, City University of Hong Kong, Shenzhen, China \\ * Corresponding author. Email: binliu9-c@my.cityu.edu.hk
}

\begin{abstract}
This paper develops a maintenance policy for a multi-component system subject to hidden failures. Components of the system are assumed to suffer from hidden failures, which can only be detected at inspection. The objective of the maintenance policy is to determine the inspection intervals for each component such that the long-run cost rate is minimized. Due to the dependence among components, an exact optimal solution is difficult to obtain. Concerned with the intractability of the problem, a heuristic method named "base interval approach" is adopted to reduce the computational complexity. Performance of the base interval approach is analyzed and the result shows that the proposed policy can approximate the optimal policy within a small factor. Two numerical examples are presented to illustrate the effectiveness of the policy.
\end{abstract}

Key words: Hidden failure, multi-component system, maintenance optimization, inspectionreplacement policy, base interval approach

\section{Introduction}

With the integration of technologies, modern systems are often complex systems consisting of multiple components. For multi-component systems, interactions among components cannot be 
neglected when making maintenance decisions. Optimal maintenance strategy for an individual component may not necessarily remain optimal for a system consisting of multiple dependent components. If no dependence exists among components, maintenance strategy for multicomponent systems is identical with that for a single-component systems (Wang, 2002). Dependency among components however brings a lot of challenges to multi-component maintenance optimization (Gustavsson et al, 2014; Phan \& Zhu, 2015; Liu et al, 2015).

In the literature, two strategies have been widely used to deal with the multi-component maintenance issue, namely, group maintenance and opportunistic maintenance (Nicolai \& Dekker, 2008). Group maintenance implies that multiple components are replaced in groups at predetermined times (Xiao et al, 2016). Vu et al (2014) proposed a dynamic group maintenance strategy for multi-component systems. Gao et al (2015) proposed a dynamic interval maintenance policy, which is more effective in reducing unnecessary maintenance actions compared with periodic preventive maintenance. Opportunistic maintenance occurs in the case that components are maintained when a maintenance opportunity arises, e.g., unexpected system shutdown. Pandey et al (2016) proposed a preventive maintenance scheduling model where maintenance actions are implemented during system breakdown. Recently, more advanced maintenance policies (e.g., condition-based maintenance) have been applied for multi-component systems (Camci, 2009). Liu et al (2014) proposed a value-based maintenance policy for multicomponent systems with degrading components. In the work of Tian \& Liao (2011), a condition based maintenance strategy with proportional hazards model was developed for multi-component systems.

Although numerous maintenance policies for multi-component systems have been proposed in literature, most of the models focus on catastrophic or sudden failures (failures that are selfannouncing). However, in many cases, failures are not self-announcing, that a failure remains dormant before being exposed by inspection techniques or other system disturbances. This type of failure is referred to as hidden failure or soft failure (Ye et al, 2014; Ye \& Xie, 2015; Wang et al, 2016). Hidden failure itself does not lead to system breakdown, but the existence of hidden failure may cause performance loss during system operation. Hidden failure is very common in modern systems, particularly in stand-by redundant systems and protection systems (Xiao \& Ye, 2016). Yang et al (2006) reported that hidden failure in protection system may cause multiple 
component outages and even spread power system disturbances. Cheng et al (2013) pointed out that hidden failure of relay protection system is the main contributor of cascading failures and blackouts.

A common practice to deal with hidden failure is to implement an inspection policy to detect the existence of hidden failures (Lam \& Banjevic, 2015). Based on the interval between two consecutive inspections, inspection policy can be classified into two types: periodic inspection and sequential inspection (non-periodic). A common practice in industry is to implement periodic inspection at constant intervals, due to its feasibility and applicability. In literature, there exist plentiful research works on dealing with hidden failures. Wang \& Pham (2011) investigated a multi-objective maintenance optimization for systems with dependent competing risks of degradation wear and random shocks. Ahmadi \& Kumar (2011) studied cost based risk of system with hidden failures, where the optimal inspection and restoration intervals are determined based on the cost criterion. He et al (2015) considered an imperfect inspection policy for system subject to hidden failures, in which the interval of imperfect inspection interval and the number of imperfect inspections are determined so as to achieve an optimal maintenance strategy. Liu et al (2017) proposed a condition-based maintenance policy for a system with age- and statedependent operating cost, where the side effect of degradation is investigated.

Although numerous studies have accommodated to hidden failures, most focus on maintenance/inspection policy for a single-component system. However, modern systems are usually subject to multiple hidden failures. For example, a protection system contains multiple components such as transducer and relays, and hidden failure may occur in any of the constituents. It is thus necessary to investigate hidden failure in a multi-component context. Yet few efforts have been devoted to multiple-component maintenance policy with hidden failures. Zhu et al (2016) established a multi-level maintenance model for a system with multiple failure modes. Taghipour \& Banjevic (2011) developed a periodic inspection policy for a repairable system, where the components are subject to hidden failures. In that work, the optimal inspection interval was applied for all the components. Huynh et al (2014) proposed a multi-level maintenance policy, where the system was intervened at both the component and the system level. Long-run cost rate was minimized by jointly optimizing the inspection interval and preventive maintenance thresholds. One deficiency of the above methods is that all the 
components are inspected simultaneously or a common metric is used for all the components. However, as the components in the system vary in degradation process and maintenance cost, it is more desirable to apply different maintenance metrics for heterogeneous components.

In this paper, we aim to develop a maintenance policy for a multi-component system subject to hidden failures. The objective of this study is to determine the inspection intervals for each component such that the long-run cost rate is minimized. Here, we focus on the effect of economic dependence since economic dependence is the most common interaction in a multicomponent system. With respect to the maintenance optimization of the multi-component system, the difficulty lies in the computational complexity. Actually, optimization of multi-component maintenance scheduling with economic dependence has been proven to be an NP-complete problem (Levi et al, 2014). To deal with the intractability of this problem, we adopt a heuristic method named "base interval approach" on the basis of Laggoune et al (2009) and Hopp \& Kuo (1998). The base interval approach reschedules the inspection interval of each component as an integer multiplier of a base interval, so as to share the common downtime cost and reduce maintenance cost. In addition, we investigate the performance of the base interval approach, where the effectiveness of proposed approach is demonstrated both analytically and numerically.

The remainder of this paper is organized as follows. Section 2 describes the problem and formulates the maintenance model. Maintenance policy using base interval approach is proposed in Section 3 and performance analysis of the approach is presented in Section 4. Numerical examples are presented in Section 5. Finally, concluding remarks and future discussions are given in Section 6.

\section{Notations}

$\begin{array}{ll}C_{I} & \text { Common downtime cost due to inspection } \\ c_{d}^{i} & \text { Downtime cost per unit time of component } i \\ c_{I}^{i} & \text { Inspection cost of component } i \\ c_{r}^{i} & \text { Replacement cost of component } i \\ T_{d}^{i}(\mathrm{t}) & \text { Downtime of component } i \text { till time } t\end{array}$




$\begin{array}{ll}G(\bullet) & \text { Long-run cost rate of the system } \\ G_{i}(\bullet) & \text { Long-run cost rate of component } i \text {, without considering the common cost } \\ k_{i} & \text { Integer multiplier, indicating the rescheduled inspection interval of component } i \\ i=1,2, \ldots, n & \text { Component index } \\ N_{d}(\mathrm{t}) & \text { Number of system shutdown due to inspection till time } t \\ N_{I}^{i}(\mathrm{t}) & \text { Number of inspections of component } i \text { till time } t \\ n & \text { Number of components } \\ R_{i}(t) & \text { Reliability function of component } i \text { at time t } \\ S_{i} & \text { Length of a renewal cycle of component } i \\ T_{i} & \text { Time to failure of component } i \\ \mu_{i} & \text { Mean time to failure of component } i \\ \tau & \text { Base inspection interval } \\ \tau_{i} & \text { Inspection interval of component } i\end{array}$

\section{Maintenance model formulation}

2.1 General description and assumptions

Consider a he system consisting of multiple non-identical components. Each component suffers an underlying deteriorating process and fails randomly according to specific lifetime distribution. The components are non-repairable; if a component is found to have failed, it has to be replaced by a new one. Note that although the components are restored to the 'as-good-asnew' condition after replacement, the whole system is not perfectly maintained except that all the components are replaced simultaneously.

An inspection-replacement policy is implemented on each component. The components are inspected at periodic intervals with the individual inspection cost $c_{I}^{i}$. When an inspection is performed on a component, the whole system has to be shut down, which incurs a common downtime cost $C_{I}$. The common cost due to inspection can be shared when multiple inspections are carried out simultaneously. Without loss of generality, it is assumed that the set-up cost and system shutdown cost due to component replacement are included in $C_{I}$. Once a failure is 
detected at inspection, the associated component is replaced immediately. During the period of hidden failures, additional cost is incurred due to performance loss of the component, denoted as $c_{d}^{i}$.

In terms of constructing a realistic model, the following assumptions are made:

1. Failure of a component is not self-announcing; it can only be revealed at inspection.

2. Inspection can be implemented on each component; inspection on a component is perfect. When a failure occurs, it can always be discovered at the next inspection.

3. A common cost is incurred at each inspection time, which can be shared when multiple inspections are carried out simultaneously. The assumption of constant common cost is reasonable when multiple maintenance crews are available to inspect the components.

4. Only economic dependence exists between components. Structural dependence and stochastic dependence are not considered.

5. The component is non-reparable; only replacement is applicable when a failure is revealed on a component. Replacement is instantaneous and restores the component to the 'as-good-as-new' condition.

6. Deterioration of a component remains unchanged during inspection.

\subsection{Cost model}

Consider a system consisting of $n$ heterogeneous components. Let $\tau_{i}, i=1,2, \ldots, n$, be the inspection intervals for component $i$. The cost items can be divided into two categories. One is the component-level cost, which is related to specific component and varies for different components. The other category is the system-level cost, which is irrelevant to the component and can be shared by multiple maintenance actions.

Component-level cost: component inspection $\operatorname{cost} c_{I}^{i}$, component replacement $\operatorname{cost} c_{r}^{i}$, performance loss (due to hidden failures of components) cost per unit time $c_{d}^{i}$.

System-level cost: common downtime cost due to inspection $C_{I}$.

For an individual component $i$, one has to pay the associated cost at time $t$,

$$
\tilde{H}_{i}\left(t ; \tau_{i}\right)=\left(C_{I}+c_{I}^{i}\right) N_{I}^{i}\left(t ; \tau_{i}\right)+c_{r}^{i} N_{r}^{i}\left(t ; \tau_{i}\right)+c_{d}^{i} T_{d}^{i}\left(t ; \tau_{i}\right)
$$


where $\tau_{i}$ is the inspection interval of component $i, \tilde{H}_{i}$ is the total cost of component $i$ incorporating the common downtime $\operatorname{cost}, N_{I}^{i}$ is the cumulative number of inspections for component $i, N_{r}^{i}\left(t ; \tau_{i}\right)$ is the cumulative number of replacement for component $i$, and $T_{d}^{i}\left(t ; \tau_{i}\right)$ is the cumulative downtime of component $i$. However, for a multi-component system, there may be cases that the common cost has already been shared by other components (e.g., due to inspection of other components). If this happens all the time, then we can neglect the common $\operatorname{cost} C_{I}$. In such cases, the corresponding cost of a component at time $t$ is

$$
H_{i}\left(t ; \tau_{i}\right)=c_{I}^{i} N_{I}^{i}\left(t ; \tau_{i}\right)+c_{r}^{i} N_{r}^{i}\left(t ; \tau_{i}\right)+c_{d}^{i} T_{d}^{i}\left(t ; \tau_{i}\right)
$$

As shown in Eq (1) and (2), the total maintenance cost can be reduced if multiple components are inspected simultaneously. As the common cost can be shared when performing inspections together, it is of interest to schedule inspections in groups so that multiple inspections can be implemented at one scheduled inspection time. However, on the other hand, rescheduling the inspection time incurs additional cost. As we will see in the following sections, each component has its optimal inspection interval so as to minimize the individual long-run cost rate. If the inspection interval is postponed or advanced in order to share common downtime cost $C_{I}$, additional cost is incurred compared with the minimal cost obtained at the optimal inspection interval. We have to consider this tradeoff when grouping multiple components to share the common downtime cost. An optimal policy should balance the potential benefit and the associated additional cost.

If the components are inspected with intervals $\tau_{1}, \tau_{2}, \ldots, \tau_{n}$, the long-run cost rate of the system is expressed as

$$
G^{\left(\tau_{1}, \tau_{2}, \ldots, \tau_{n}\right)}=\lim _{t \rightarrow \infty} \frac{\sum_{i=1}^{n} H_{i}\left(t ; \tau_{i}\right)+N_{d}\left(t ; \tau_{1}, \tau_{2}, \ldots, \tau_{n}\right) C_{I}}{t}
$$

where $N_{d}(t)$ is the number of system shutdown till time $t$. Obtaining an optimal solution of Eq (3) is difficult due to the economic dependence among components. Indeed, optimization of Eq (3) is an NP-hard problem as the number of combinations increases exponentially with the number of components. Due to the intractability of this problem, we have to resort to heuristic methods 
that can provide a near optimal solution. In the following, we adopt base interval approach to deal with the computational complexity.

\section{Maintenance policy with base interval approach}

Base interval approach was initially proposed to solve the joint replacement issue of an aircraft engine (Hopp and Kuo, 1998). It was inspired by the power-of-two replenishment policy in inventory management (Jackson et al, 1985). Laggoune et al (2009) applied the base interval approach in opportunistic maintenance for a continuously operating system. The basic idea of base interval approach is to find a base interval $\tau$ such that the common cost is charged at every $\tau$ time units and the component is inspected at some integer multipliers of the base interval, $k_{i} \tau$. In other words, at least one component is inspected in each multiple of $\tau$. Under this scenario, our objective is to find the optimal base interval $\tau$ and a set of integers $\left\{k_{i}, i=1,2, \ldots, n\right\}$ such that the long-run cost rate of the system is minimized. The optimization problem is formulated into

$$
\begin{gathered}
\min _{\tau, k_{1}, k_{2}, \ldots, k_{n}} \lim _{t \rightarrow \infty} \frac{\sum_{i=1}^{n} H_{i}\left(t ; k_{i} \tau\right)}{t}+\frac{C_{I}}{\tau} \\
\text { s.t. } \quad \tau>0, k_{i} \in Z^{+}, \quad \forall i=1,2, \ldots, n
\end{gathered}
$$

where $Z^{+}$denotes the set of positive integers.

A natural way to calculate the long-run cost rate is to use the renewal cycle theorem and compute the expected cost rate in a renewal cycle of the system (Zhang et al, 2015). However, in the present case, a renewal cycle occurs only when all the components are replaced simultaneously, which takes extremely long time or even never occurs. Hence, it is difficult or even impossible to compute the long-run cost rate function based on renewal cycle theorem. However, by observing the cost structure of $\mathrm{Eq}$ (4), we can decompose the objective function from system level into component level, and then apply the renewal cycle theorem individually.

As we can see, the objective function contains two terms: the first term, $\lim _{t \rightarrow \infty} \sum_{i=1}^{n} H_{i}\left(t ; k_{i} \tau\right) / t$, is a summation of individual cost, while the second term, $C_{I} / \tau$, is irrelevant to individual 
component. By taking advantage of the cost structure, we proceed to study the individual optimal maintenance policy so as to reduce the computational complexity.

\subsection{Individual optimal maintenance policy}

In this section, we formulate a cost model for an individual component and obtain the optimal individual inspection interval. When a component is replaced at inspection, the component is restored to the 'as-good-as-new' state, which constitutes a renewal cycle (Liu et al, 2016). According to the renewal cycle theorem, the individual long-run cost rate is given as

$$
G_{i}\left(\tau_{i}\right)=\lim _{t \rightarrow \infty} \frac{H_{i}\left(t ; \tau_{i}\right)}{t}=\frac{E\left[H_{i}\left(S_{i}\right)\right]}{E\left[S_{i}\right]}
$$

where $S_{i}$ is the length of a renewal cycle of component $i$. Denote $T_{i}$ as the failure time of component $i$. Let $R_{i}(t)$ be the reliability function of the component and $F_{T_{i}}(t)$ be the cumulative density function (cdf) of the failure time. For notational simplicity, we denote the number of inspection in a renewal cycle and the downtime in a renewal cycle for component $i$ as $N_{I}^{i}$ and $T_{d}^{i}$, instead of $N_{I}^{i}\left(S_{i}\right)$ and $T_{d}^{i}\left(S_{i}\right) . N_{I}^{i}$ is related to the failure time and the inspection interval, which is denoted as $N_{I}^{i}=\left\lceil T_{i} / \tau_{i}\right\rceil$ (Badia et al, 2001). The expectation of $N_{I}^{i}$ is calculated as

$$
\begin{aligned}
& E\left[N_{I}^{i}\right]=\sum_{j=1}^{\infty} j P\left\{(j-1) \tau_{i}<T_{i} \leq j \tau_{i}\right\} \\
& =\sum_{j=1}^{\infty} j\left(F_{T_{i}}\left(j \tau_{i}\right)-F_{T_{i}}\left((j-1) \tau_{i}\right)\right)=\sum_{j=0}^{\infty} R_{i}\left(j \tau_{i}\right)
\end{aligned}
$$

The length of a renewal cycle depends on $N_{I}^{i}$ and $\tau_{i}$, according to the assumption that replacement can only be carried out at inspection. We have $S_{i}=N_{I}^{i} \tau_{i}$ and the associated expectation $E\left[S_{i}\right]=\tau_{i} \sum_{j=0}^{\infty} R_{i}\left(j \tau_{i}\right)$ (Peng et al, 2010). The component downtime is the period between occurrence of a failure and replacement (end of a renewal cycle), $T_{d}^{i}=N_{I}^{i} \tau_{i}-T_{i}$. The expectation is $E\left[T_{d}^{i}\right]=\tau_{i} \sum_{j=0}^{\infty} R_{i}\left(j \tau_{i}\right)-\mu_{i}$, where $\mu_{i}$ is the mean time to failure (MTTF) of the component. Based on the above discussion, the expected cost in a renewal cycle can be obtained as 


$$
E\left[H_{i}\left(S_{i}\right)\right]=c_{d}^{i}\left(\tau_{i} \sum_{j=0}^{\infty} R_{i}\left(j \tau_{i}\right)-\mu_{i}\right)+c_{r}^{i}+c_{I}^{i} \sum_{j=0}^{\infty} R_{i}\left(j \tau_{i}\right)
$$

The long-run cost rate is then formulated as

$$
G_{i}\left(\tau_{i}\right)=\frac{E\left[H_{i}\left(S_{i}\right)\right]}{E\left[S_{i}\right]}=c_{d}^{i}+\frac{-c_{d}^{i} \mu_{i}+c_{r}^{i}+c_{I}^{i} \sum_{j=0}^{\infty} R_{i}\left(j \tau_{i}\right)}{\tau_{i} \sum_{j=0}^{\infty} R_{i}\left(j \tau_{i}\right)}
$$

Proposition 1. If $c_{d}^{i} \mu_{i}-c_{r}^{i}>c_{I}^{i}$, there exists optimal inspection interval $\tau_{i}^{*}$ such that the longrun cost rate reaches its minimum. Otherwise, $\tau_{i}^{*}=\infty$. Mathematically,

$$
\left\{\begin{array}{l}
\tau_{i}^{*} \in\left\{\tau_{i}: d G_{i}\left(\tau_{i}\right) / d \tau_{i}=0\right\}, \text { if } c_{d}^{i} \mu_{i}-c_{r}^{i}>c_{I}^{i} \\
\tau_{i}^{*}=\infty, \text { otherwise }
\end{array}\right.
$$

The detailed proof is shown in the Appendix.

Proposition 1 implies that if $c_{d}^{i} \mu_{i}-c_{r}^{i}>c_{I}^{i}$, it is beneficial to inspect the system with the inspection interval $\tau_{i}^{*}$; otherwise, inspection is unprofitable and we should resort to other maintenance policies. In this study, we consider the case that the condition of $c_{d}^{i} \mu_{i}-c_{r}^{i}>c_{I}^{i}$ can always be satisfied; otherwise, the maintenance problem is trivial and makes no sense. This assumption is plausible as the downtime cost is relatively high for modern complex systems and the inspection cost is becoming lower with the development of inspection techniques. The condition in Proposition 1 is equivalent to $\mu_{i}>\left(c_{I}^{i}+c_{r}^{i}\right) / c_{d}^{i}$, which implies that whether an optimal inspection interval exists or not can be easily judged by comparing the MTTF of a component with a constant value.

\subsection{Selection of $k_{i}$}

Based on the previous discussions, we can sketch $G_{i}\left(\tau_{i}\right)$ as shown in Fig 1. Since the system is inspected at some integer multiples of the base interval $\tau$, the optimal $k_{i}$ should be either $\left\lfloor\tau_{i}^{*} / \tau\right\rfloor$ or $\left\lceil\tau_{i}^{*} / \tau\right\rceil$, depending on which is more cost-effective. $k_{i}$ can be expressed as 


$$
k_{i}=\left\{\begin{array}{l}
\left\lfloor\tau_{i}^{*} / \tau\right], \text { if } \frac{-c_{d}^{i} \mu_{i}+c_{r}^{i}+c_{I}^{i} \sum_{j=0}^{\infty} R_{i}\left(j\left\lfloor\tau_{i}^{*} / \tau\right\rfloor \tau\right)}{\left\lfloor\tau_{i}^{*} / \tau\right\rfloor \tau \sum_{j=0}^{\infty} R_{i}\left(j\left\lfloor\tau_{i}^{*} / \tau\right] \tau\right)}<\frac{-c_{d}^{i} \mu_{i}+c_{r}^{i}+c_{I}^{i} \sum_{j=0}^{\infty} R_{i}\left(j\left\lceil\tau_{i}^{*} / \tau\right\rceil \tau\right)}{\left\lceil\tau_{i}^{*} / \tau\right\rceil \tau \sum_{j=0}^{\infty} R_{i}\left(j\left\lceil\tau_{i}^{*} / \tau\right\rceil \tau\right)} \\
\left\lceil\tau_{i}^{*} / \tau\right\rceil, \text { otherwise }
\end{array}\right.
$$

Therefore, once the base interval is given, the optimal inspection intervals for each component can be easily obtained. The objective is reduce to find the optimal base interval $\tau^{*}$ such that the long-run cost rate of the system, $G(\tau)$, is minimized, i.e.,

$$
\min _{\tau>0} G(\tau)=\frac{C_{I}}{\tau}+\sum_{i=1}^{n} c_{d}^{i}+\sum_{i=1}^{n}\left[\frac{-c_{d}^{i} \mu_{i}+c_{r}^{i}+c_{I}^{i} \sum_{j=0}^{\infty} R_{i}\left(j\left\lfloor\tau_{i}^{*} / \tau\right\rfloor \tau\right)}{\left\lfloor\tau_{i}^{*} / \tau\right\rfloor \tau \sum_{j=0}^{\infty} R_{i}\left(j\left\lfloor\tau_{i}^{*} / \tau\right\rfloor \tau\right)} \wedge \frac{-c_{d}^{i} \mu_{i}+c_{r}^{i}+c_{I}^{i} \sum_{j=0}^{\infty} R_{i}\left(j\left\lceil\tau_{i}^{*} / \tau\right\rceil \tau\right)}{\left\lceil\tau_{i}^{*} / \tau\right\rceil \tau \sum_{j=0}^{\infty} R_{i}\left(j\left\lceil\tau_{i}^{*} / \tau\right\rceil \tau\right)}\right]
$$

where the operator $(x \wedge y)=\min (x, y)$.

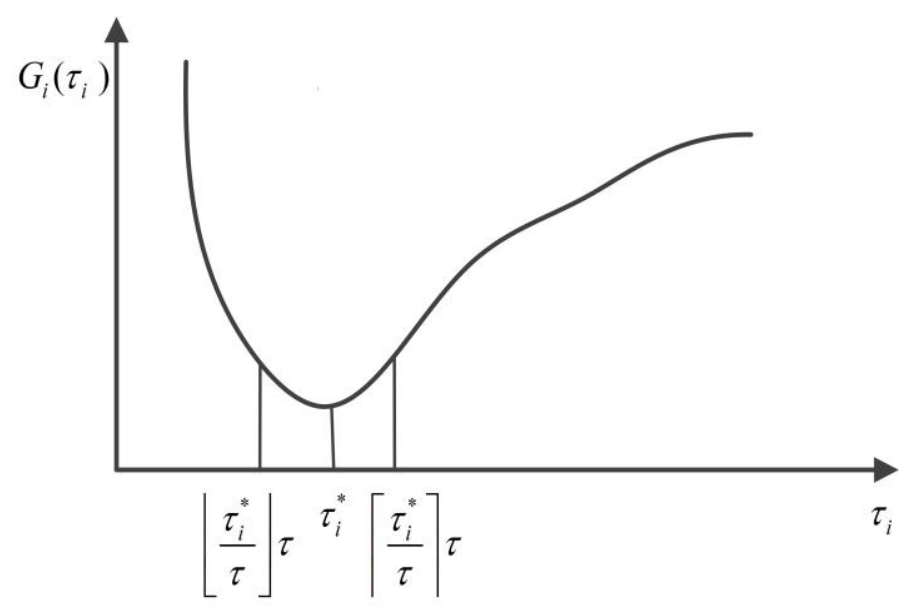

Fig 1 Sketch of $G_{i}\left(\tau_{i}\right)$

Optimal solution of Eq (7) can be obtained by a simple search algorithm, described as follows:

1. Initialization: For each component, compute the individual optimal inspection interval $\tau_{i}^{*}, i=1,2, \ldots, n$.

2. Begin with a small $\tau$. 
3. Determine $k_{i}$ for each component, according to Eq (6).

4. Compute the value of $G(\tau)$ according to $\mathrm{Eq}(7)$.

5. Increase $\tau$ for a small increment, repeat step 3-4. Stop until $\tau>\max \left\{\tau_{i}^{*}: i=1,2, \ldots, n\right\}$.

6. Output the minimal $G^{*}(\tau)$ and the associated $\tau^{*}$.

The time complexity of the algorithm is $O(n)$. Compared with the traditional approaches such as rolling horizon approach and brute-force search approach, the proposed base interval approach shows the advantage of computational effectiveness.

\section{Performance analysis}

Now that we have described the base interval policy, we proceed to analyze the effectiveness of the policy. A natural way to achieve this purpose is to compare the cost value obtained by our approach with the optimum value. However, for the present case, the optimal solution is difficult to obtain. Hence, instead, we demonstrate the effectiveness of our policy by comparing the upper bound of our approach with the lower bound of the optimal solution.

\subsection{Lower bound of the optimal solution}

The idea of obtaining a lower bound is to allocate the maintenance cost of the whole system on individual components. Let $\left\{t_{l}, l=1,2, \ldots\right\}$ denote the time when the system is shut down due to inspection, where $l$ is the number index of system shutdown. Let $x_{i}^{t_{l}}$ be the action for component $i$ at time $t_{l}$, where

$$
x_{i}^{t_{l}}=\left\{\begin{array}{l}
0, \text { if no inspection is performed for component } i \text { at } t_{l} \\
1, \text { if inspection is performed for component } i \text { at } t_{l}
\end{array}\right.
$$

Let $\delta(\bullet)$ be the indicator function, where for any $\alpha \geq 0$,

$$
\delta(\alpha)=\left\{\begin{array}{l}
0, \text { if } \alpha=0 \\
1, \text { if } \alpha>0
\end{array}\right.
$$

With the notations defined above, we can transform the problem of minimizing system longrun cost rate into finding the optimal $x_{i}^{t_{i} *}, l=1,2, \ldots, i=1,2, \ldots, n$, that minimize 


$$
\min _{x_{i}^{t_{l}}} \mathrm{Z}=\lim _{t \rightarrow \infty} \frac{\sum_{i=1}^{n} H_{i}\left(t ; \tilde{\tau}_{i}\right)+\delta\left(\sum_{i=1}^{n} x_{i}^{t_{l}}\right) C_{I}}{t}
$$

It is straightforward to have (Atkins \& Iyogun, 1987)

$$
\delta\left(\sum_{i=1}^{n} x_{i}^{t_{l}}\right) \geq \sum_{i=1}^{n} \eta_{i} \delta\left(x_{i}^{t_{l}}\right)
$$

where $\eta_{i} \geq 0, \forall i=1,2, \ldots, n$, and $\sum_{i=1}^{n} \eta_{i}=1$. Then, the lower bound of $\mathrm{Z}$ can be readily obtained as

$$
\begin{aligned}
& \underline{\mathrm{Z}}=\lim _{t \rightarrow \infty} \frac{\sum_{i=1}^{n} H_{i}\left(t ; \tilde{\tau}_{i}\right)+\sum_{i=1}^{n} \eta_{i} \delta\left(x_{i}^{t_{l}}\right) C_{I}}{t} \\
& =\sum_{i=1}^{n} \lim _{t \rightarrow \infty} \frac{H_{i}\left(t ; \tilde{\tau}_{i}\right)+\eta_{i} \delta\left(x_{i}^{t_{l}}\right) C_{I}}{t}
\end{aligned}
$$

For notational simplicity, denote

$$
\mathrm{Z}_{i}\left(\eta_{i}, \tilde{\tau}_{i}\right)=\lim _{t \rightarrow \infty} \frac{H_{i}\left(t ; \tilde{\tau}_{i}\right)+\eta_{i} \delta\left(x_{i}^{t_{l}}\right) C_{I}}{t}
$$

so that $\underline{Z}=\sum_{i=1}^{n} Z_{i}\left(\eta_{i}, \tilde{\tau}_{i}\right)$. Eq (9) implies that the lower bound $\underline{Z}$ can be decomposed into $n$ single-unit cost items, with the common constraints $\sum_{i=1}^{n} \eta_{i}=1$ and $\eta_{i} \geq 0$. For component $i$, at each inspection time, the individual inspection cost $c_{I}^{i}$ and common shutdown $\operatorname{cost} \eta_{i} C_{I}$ are charged; at the end of a renewal cycle of the component, the individual replacement cost $c_{r}^{i}$ and component downtime cost $c_{d}^{i} \cdot T_{d}^{i}$ are incurred. Therefore, for a fixed $\eta_{i}, \mathrm{Z}_{i}\left(\eta_{i}, \tilde{\tau}_{i}\right)$ is equivalent to the individual long-run cost rate of component $i$, with additional common downtime $\operatorname{cost} \eta_{i} C_{I}$ at each inspection. The optimal inspection interval, $\tilde{\tau}_{i}^{*}\left(\eta_{i}\right)$, is obtained as 


$$
\begin{aligned}
& \tilde{\tau}_{i}^{*}\left(\eta_{i}\right)=\arg \min _{\tilde{\tau}_{i}} Z_{i}\left(\eta_{i}, \tilde{\tau}_{i}^{*}\right) \\
& =\arg \min _{\tilde{\tau}_{i}} c_{d}^{i}+\frac{-c_{d}^{i} \mu_{i}+c_{r}^{i}+\left(c_{I}^{i}+\eta_{i} C_{I}\right) \sum_{j=0}^{\infty} R_{i}\left(j \tilde{\tau}_{i}\right)}{\tilde{\tau}_{i} \sum_{j=0}^{\infty} R_{i}\left(j \tilde{\tau}_{i}\right)}
\end{aligned}
$$

Eq (10) implies that if $\eta_{i} \geq 0$ is given for component $i$, an optimal inspection interval $\tilde{\tau}_{i}^{*}\left(\eta_{i}\right)$ can be readily obtained. Let $Z_{i}^{*}\left(\eta_{i}\right)=\left.Z_{i}\left(\eta_{i}, \tilde{\tau}_{i}\right)\right|_{\tilde{\tau}_{i} \in \tilde{\tau}_{i}^{*}\left(\eta_{i}\right)}$, the tightest lower bound becomes

$$
\begin{gathered}
\max _{\eta_{i}} \underline{\mathrm{Z}}=\sum_{i=1}^{n} \mathrm{Z}_{i}^{*}\left(\eta_{i}\right) \\
\text { s.t. } \sum_{i=1}^{n} \eta_{i}=1, \eta_{i} \geq 0, \forall i=1,2, \ldots, n
\end{gathered}
$$

Based on the above discussions, the optimization problem in Eq (8) is transformed into finding optimal allocation $\left(\eta_{i} C_{I}\right)$ of the common downtime cost to each component so that the lower bound reaches its maximum. In the work of Hopp \& Kuo (1998), it was observed that the lower bound increases when allocating higher percentages of the system intervention cost to components with smaller replacement interval. Here, we follow the similar procedure and assign higher proportion of the common cost to components that are inspected more frequently.

Without loss of generality, relabel the number of components according to their individual optimal inspection intervals. Component with a smaller inspection interval is associated with a smaller index. Assuming that the common downtime cost $C_{I}$ is allocated to components $1,2, \ldots, \kappa$, with the corresponding fraction $\eta_{i}$, where $\sum_{i=1}^{\kappa} \eta_{i}=1$. The inspection interval of component $i$ is $\tau$ for $i=1,2, \ldots, \kappa$ and $\tau_{i}^{*}$ for $i=\kappa+1, \kappa+2, \ldots, n$. Then, the lower bound of the system's long-run cost rate is given as

$$
L B=\frac{C_{I}}{\tau}+\sum_{i=1}^{\kappa} G_{i}(\tau)+\sum_{i=\kappa+1}^{n} G_{i}\left(\tau_{i}^{*}\right)
$$

4.2 Upper bound of the base interval policy 
Followed by the above discussions, we first analyze the upper bound of the base interval policy for an individual component, as shown in Lemma 1.

Lemma 1. For an individual component, the base interval policy for component $i$ is guaranteed to have a cost at most $\left(\mu_{i}+\tau_{i}^{*}\right) / \mu_{i}$ times of the optimal solution.

The detailed proof is shown in the Appendix. Generally, the optimal individual inspection interval $\tau_{i}^{*}$ is much less than the MTTF of the component $\mu_{i}$ (as shown in the numerical examples), which indicates that the proposed base interval policy can perform quite well individually. Based on the individual analysis from Lemma 1, we can conclude the upper bound of the system long-run cost rate, as shown in Proposition 2.

Proposition 2. The upper bound of the base interval policy is at most $\sup \left\{\left(\mu_{i}+\tau_{i}^{*}\right) / \mu_{i}: i=1,2, \ldots, n\right\}$ times of the lower bound.

The detailed proof is shown in the Appendix. Proposition 2 indicates that the base interval policy is guaranteed to approximate the optimal solution within a small factor.

For the problem of multi-component maintenance with hidden failures, the most intuitive approaches are the individual inspection policy and common inspection policy (Taghipour \& Banjevic, 2011). It is interesting to compare the performance of the base interval approach and the extant two approaches. Obviously, the base interval approach always outperforms the common inspection policy. This is due to the fact that the base interval approach is actually a generalization of the common inspection policy. For any determined base interval $\tau$, if all the $k_{i}$ are set to 1 , then the base interval approach is reduced to the common inspection policy.

On the other hand, the advantage of base interval approach over the individual inspection policy highly depends on the common downtime cost $C_{I}$. If the common downtime cost is low, the additional cost of rescheduling the inspection intervals may overwhelm the shared cost, which implies the disadvantage of the base interval approach. This leads to another interesting issue that under what condition the base interval approach outperforms the individual inspection policy. In addition, for some components, the penalty cost due to rescheduling may exceed the common downtime cost, though not usual in reality, then it would be optimal to remain the inspection interval identical to that of the individual inspection policy, while the inspection 
intervals of other components are rescheduled. The proposed base interval approach can be further improved by determining which components should be rescheduled and which should remain unchanged.

\section{Numerical examples}

In this section, two examples are presented to illustrate the effectiveness of the base interval approach. For the first example, we consider a 3-component system, where each component follows an exponential lifetime distribution. In the second example, the proposed maintenance policy is applied in a centrifugal compressor of a catalytic reforming unit.

\subsection{An illustrative example with a 3-component system}

A system consisting of three components is used to illustrate the base interval approach. The components are assumed to follow an exponential lifetime distribution, with the reliability function $R_{i}(t)=\exp \left(-\lambda_{i} t\right)$, where $\lambda_{i}$ is the failure rate of component $i, i=1,2,3$. At first, we analyze the optimal inspection policy for an individual component. Based on the discussion in Section 3.1, we have

$$
E\left[N_{I}^{i}\right]=\sum_{j=0}^{\infty} R_{i}\left(j \tau_{i}\right)=\frac{e^{\lambda_{i} \tau_{i}}}{e^{\lambda_{i} \tau_{i}}-1}
$$

By substituting Eq (11) into Eq (5), long-run cost rate is expressed as

$$
G_{i}(\tau)=\frac{E\left[H_{i}\left(S_{i}\right)\right]}{E\left[S_{i}\right]}=c_{d}^{i}-\frac{c_{r}^{i}-\frac{c_{d}^{i}}{\lambda_{i}}}{e^{\lambda_{i} \tau_{i}} \tau_{i}}+\frac{c_{I}^{i}+c_{r}^{i}-\frac{c_{d}^{i}}{\lambda_{i}}}{\tau_{i}}
$$

The optimal inspection can be obtained by setting the derivative of to 0 , i.e.,

$$
\frac{\partial G_{i}\left(\tau_{i}\right)}{\partial \tau_{i}}=\frac{\left(c_{r}^{i}-\frac{c_{d}^{i}}{\lambda_{i}}\right)\left(\lambda_{i} \tau_{i}+1\right)}{e^{\lambda_{i} \tau_{i}} \tau_{i}^{2}}-\frac{c_{I}^{i}+c_{r}^{i}-\frac{c_{d}^{i}}{\lambda_{i}}}{\tau_{i}^{2}}=0
$$

The individual optimal inspection interval can be obtained by solving Eq (13). After some simplifications, we have 


$$
\tau_{i}^{*} \in\left\{\tau_{i}:\left(c_{r}^{i}-\frac{c_{d}^{i}}{\lambda_{i}}\right)\left(\lambda_{i} \tau_{i}+1\right)-\left(c_{I}^{i}+c_{r}^{i}-\frac{c_{d}^{i}}{\lambda_{i}}\right) e^{\lambda_{i} \tau_{i}}=0\right\}, \text { where } \frac{c_{d}^{i}}{\lambda_{i}}-c_{r}^{i}>c_{I}^{i}
$$

The parameters of the failure rate and cost items are listed in Table 1. By use of numerical methods, the individual optimal inspection interval is obtained as $\tau_{1}^{*}=1.19, \tau_{2}^{*}=1.39, \tau_{3}^{*}=2.26$, and the corresponding optimal individual long-run cost rate is respectively $G_{1}^{*}\left(\tau_{1}\right)=121.9$, $G_{2}^{*}\left(\tau_{2}\right)=187.9, G_{3}^{*}\left(\tau_{3}\right)=202.4$. In addition, we plot the variation of individual long-run cost rate $G_{i}\left(\tau_{i}\right)$ with respect to the individual inspection interval $\tau_{i}$, as shown in Fig 2 .

Table 1 Parameters of component-specific failure rate and cost items

\begin{tabular}{cccccc}
\hline Component & $\begin{array}{c}\text { Failure rate } \\
\lambda_{i}\end{array}$ & MTBF $\mu_{i}$ & $\begin{array}{c}\text { Replacement cost } \\
c_{r}^{i}\end{array}$ & $\begin{array}{c}\text { Inspection cost } \\
c_{I}^{i}\end{array}$ & $\begin{array}{c}\text { Downtime } \\
\operatorname{cost} c_{d}^{i}\end{array}$ \\
\hline 1 & 0.02 & 50 & 200 & 70 & 5000 \\
2 & 0.05 & 20 & 420 & 115 & 2500 \\
3 & 0.1 & 10 & 640 & 150 & 750 \\
\hline
\end{tabular}

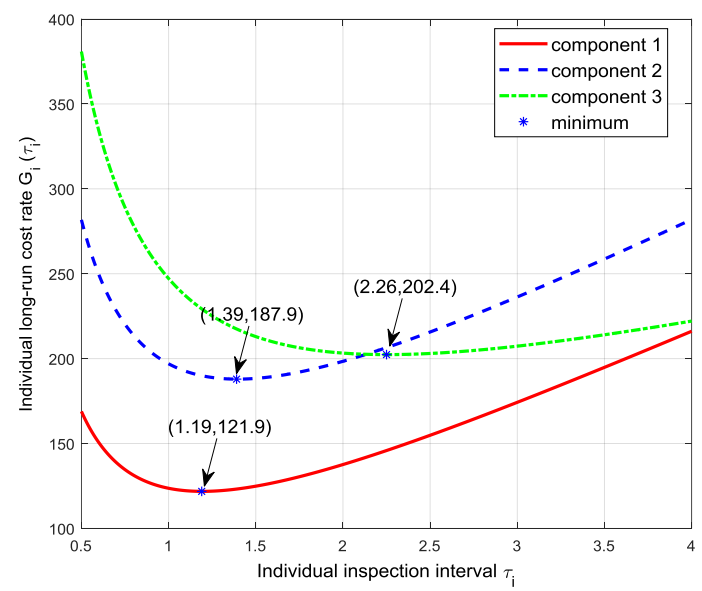

Fig 2 Variation of $G_{i}\left(\tau_{i}\right)$ with $\tau_{i}$

With regard to the whole system, the common downtime cost is set as $C_{I}=60$. The relationship between $G(\tau)$ and $\tau$ is given in Eq (7). Fig 3 shows how the long-run cost rate $G(\tau)$ changes with the base interval $\tau$. As we can see, when the base interval is $\tau=1.4$, the long-run cost rate $G(\tau)$ reaches its minimum, $G^{*}(\tau)=559.1$. The associated $k_{i}$ is given as 
$k_{1}=1, k_{2}=1, k_{3}=2$. The result implies that based on the base interval approach, when we inspect component 1 every $k_{1} \tau=1.4$ unit time, component 2 every $k_{2} \tau=1.4$ unit time, and component 3 every $k_{3} \tau=2.8$ unit time, we have the optimal maintenance policy. Table 2 shows the comparison of the base interval approach, the individual inspection policy and the common inspection policy (Taghipour \& Banjevic, 2011). The result shows that the proposed base interval approach dominates the other policies.

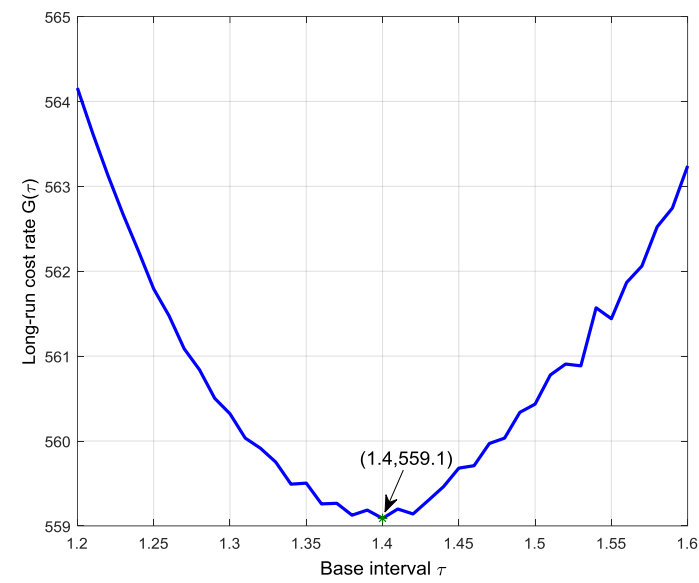

Fig 3 Variation of $G(\tau)$ with respect to $\tau$

Table 2 Comparison of the rescheduled inspection policy, the individual inspection policy and common inspection policy

\begin{tabular}{llllll}
\hline Component inspection interval & $\tau_{1}$ & $\tau_{2}$ & $\tau_{3}$ & Cost rate
\end{tabular}

\begin{tabular}{ccccc}
\hline Individual inspection policy & 1.19 & 1.39 & 2.26 & 692.2 \\
& & & & \\
\hline Common inspection policy & 1.69 & 1.69 & 1.69 & 562.9 \\
& & & & \\
\hline Rescheduled inspection policy & 1.4 & 1.4 & 2.8 & 559.1 \\
\hline
\end{tabular}


In addition, Proposition 2 implies that the upper bound of the base interval policy is at most $\sup \left\{\left(\mu_{i}+\tau_{i}^{*}\right) / \mu_{i}: i=1,2,3\right\}=1.226$ times of the lower bound. Here, the lower bound is calculated as $L B=556.4$. The long-run cost rate obtained by the base interval approach is actually 1.005 times of the lower bound, which indicates that the base interval approach performs quite effectively.

We are interested in the value of the common downtime cost $C_{I}$, since $C_{I}$ characterizes the economic dependence among components and affects the necessity of performing base interval approach. Sensitivity analysis is carried out on $C_{I}$ to find out how $C_{I}$ influences the optimal base interval and the optimal long-run cost rate. Fig 4 shows how the optimal inspection interval $\tau^{*}$ and the associated long-run cost rate $G^{*}(\tau)$ vary with different $C_{I}$. As can be observed, $G^{*}(\tau)$ increases monotonically with $C_{I}$ and $\tau^{*}$ shows a non-decreasing trend. This can be explained by the fact that the long-run cost rate $G(\tau)$ consists of two items: one is related to the common cost, and the other is determined by the individual cost rate. When the common cost $C_{I}$ increases, the cost item $C_{I} / \tau$ has an increasing effect on $G(\tau)$. Therefore, the optimal base interval $\tau$ increases with $C_{I}$ to reduce the number of system shutdowns.

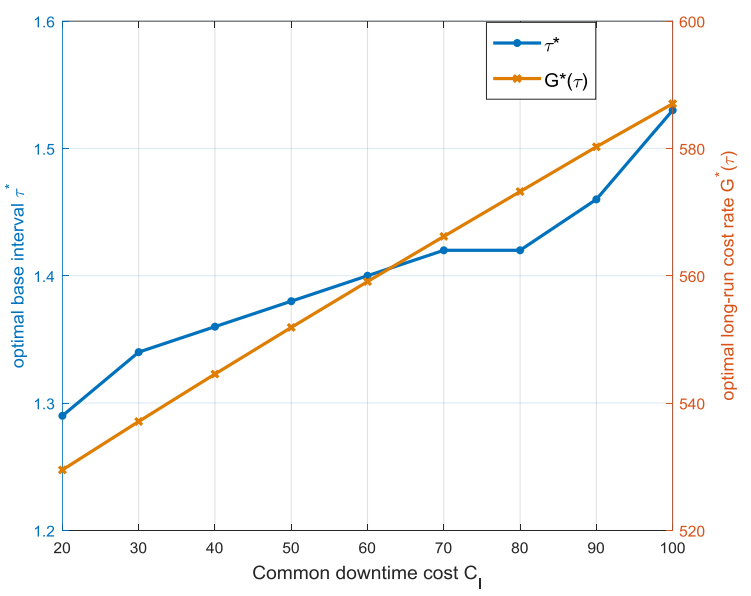

Fig 4 Sensitivity analysis on $C_{I}$ 

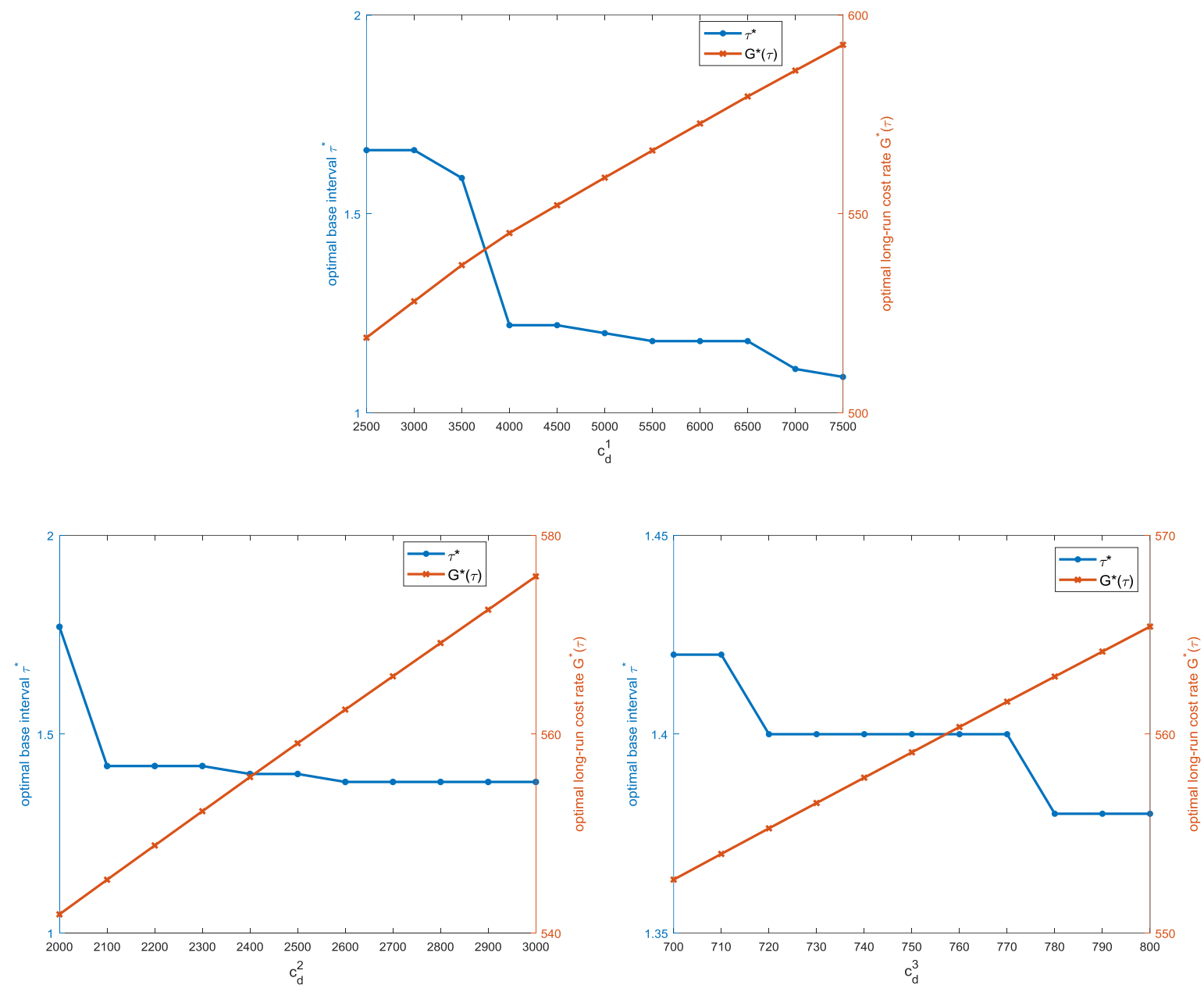

Fig 5 Variation of $\tau^{*}$ and $G^{*}(\tau)$ in terms of individual downtime $\operatorname{cost} C_{d}^{i}$

(a) component $1 C_{d}^{1}$ (b) component $2 C_{d}^{2}$ (c) component $3 C_{d}^{3}$

Additionally, Fig 5 presents the variation of $\tau^{*}$ and $G^{*}(\tau)$ in terms of the individual downtime $\operatorname{cost} C_{d}^{i}$. Obviously, the optimal long-run cost rate $G^{*}(\tau)$ shows a monotone increasing trend with $C_{d}^{i}$. The optimal inspection interval $\tau^{*}$, however, presents a non-increasing trend. This is due to the fact that a more frequent inspection takes effect in reducing the downtime cost. With the increase of the individual downtime cost, a smaller inspection interval is expected to balance the effect of increasing downtime cost. 

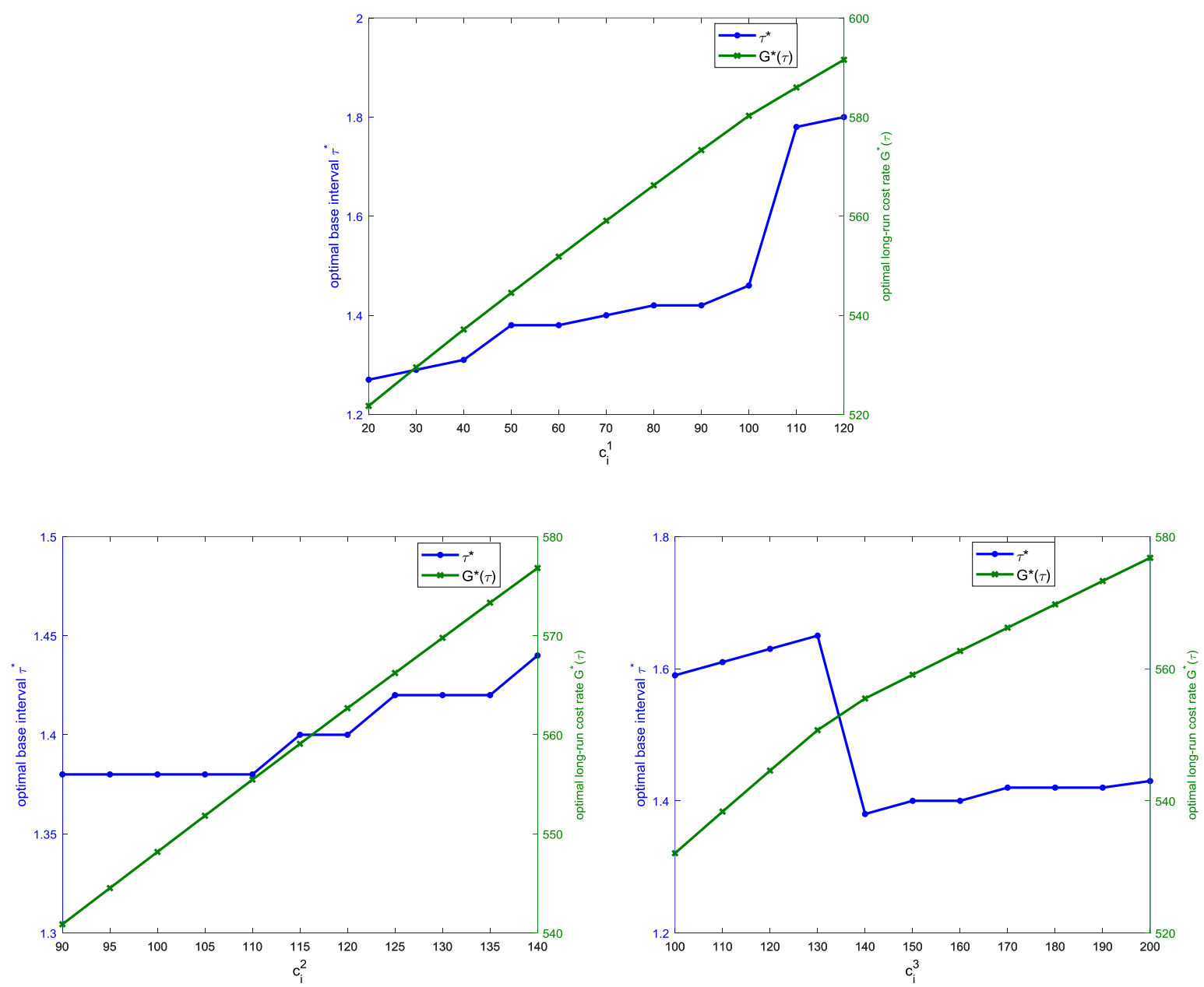

Fig 6 Variation of $\tau^{*}$ and $G^{*}(\tau)$ in terms of individual inspection $\operatorname{cost} C_{I}^{i}$

(a) component $1 C_{I}^{1}$ (b) component $2 C_{I}^{2}$ (c) component $3 C_{I}^{3}$

Fig 6 investigates the effect of individual inspection cost on the optimal maintenance policy. For component 1 and component 2, the optimal inspection interval $\tau^{*}$ shows a non-decreasing trend. The optimal maintenance policy calls for a larger inspection interval so as to balance the tradeoff of an increased inspection cost. However, component 3 shows a non-conform trend. $\tau^{*}$ increases for $C_{I}^{3} \in(100,130)$ and $C_{I}^{3} \in(140,200)$ respectively. However, there is a drop-off for $C_{I}^{3} \in(130,140)$. This is due to the fact that the integer multiplier $k_{3}$ varies for $C_{I}^{3} \in(130,140)$. In the present setting, $k_{3}$ increases from 1 to 2 when $C_{I}^{3}$ changes from 130 to 140 . Although the optimal base interval decreases, the inspection interval of component 3 still increases, due to the increase of $k_{3}$. 


\subsection{Application in refinery centrifugal compressor}

In this section, we apply the base interval inspection policy to a centrifugal compressor of a catalytic reforming unit. A centrifugal compressor is an essential constitute of a catalytic reforming unit, which plays a vital role in oil refinery. As reported in Laggoune et al (2009), a compressor dysfunction may cause pressure loss in the reforming unit and consequently lead to performance loss of the system. In addition, the centrifugal compressor is responsible for preheating and providing air to guarantee that the unit operates under proper condition. A centrifugal compressor comprises multiple components, such as sheathing, tightness and so on. Here, we assume that the components of a compressor operate in a redundant mode, where failure of a component is not self-evident. The base interval inspection policy is adopted to reduce the maintenance cost and enhance system performance.

Table 3 Component-specific Weibull parameters and cost items

\begin{tabular}{cccccccc}
\hline $\begin{array}{c}\text { Compone } \\
\text { nt }\end{array}$ & code & $\begin{array}{c}\text { Shape } \\
\text { parameter } \\
\beta_{i}\end{array}$ & $\begin{array}{c}\text { Scale } \\
\text { parameter } \\
\theta_{i}\end{array}$ & $\begin{array}{c}\text { MTBF } \\
\mu_{i}(\text { day })\end{array}$ & $\begin{array}{c}\text { Replacemen } \\
\text { t cost } c_{r}^{i}(€)\end{array}$ & $\begin{array}{c}\text { Inspection } \\
\text { cost } c_{I}^{i}(€) \\
\text { (assumed) }\end{array}$ & $\begin{array}{c}\text { Downtime } \\
\text { cost } c_{d}^{i}(€) \\
\text { (assumed) }\end{array}$ \\
\hline Sheathing & $\mathrm{C} 286$ & 1.73 & 486 & 483 & 14868 & 1200 & 350 \\
Sheathing & $\mathrm{C} 285$ & 1.88 & 507 & 475 & 39204 & 320 & 1100 \\
$\begin{array}{c}\text { Tightness } \\
\text { Stub }\end{array}$ & $\mathrm{C} 275$ & 2.43 & 286 & 240 & 44880 & 330 & 1000 \\
$\begin{array}{c}\text { bearing } \\
\text { Tightness }\end{array}$ & $\mathrm{C} 230$ & 2.53 & 898 & 787 & 57876 & 1180 & 1600 \\
$\begin{array}{c}\text { ring } \\
\text { Carrying }\end{array}$ & $\mathrm{C} 460$ & 2.14 & 905 & 844 & 73860 & 420 & 1900 \\
$\begin{array}{c}\text { bearing } \\
\text { Stub }\end{array}$ & $\mathrm{C} 419$ & 3.55 & 736 & 636 & 46752 & 360 & 1600 \\
bearing & $\mathrm{C} 401$ & 2.68 & 1094 & 888 & 48568 & 1670 & 1700 \\
$\begin{array}{c}\text { Labyrinth } \\
\text { support }\end{array}$ & $\mathrm{C} 780$ & 2.09 & 1388 & 1047 & 74232 & 1700 & 1800 \\
\hline
\end{tabular}

The compressor consists of eight components, wherein each component is associated with a code, as shown in Table 3. The components are assumed to follow a two-parameter Weibull distribution. The reliability function of component $i$ is denoted as $R_{i}(t)=\exp -\left[\left(t / \theta_{i}\right) \beta_{i}\right]$, 
where $\theta_{i}$ is the scale parameter and $\beta_{i}$ is the shape parameter. Table 3 gives the value of Weibull parameters and the associated cost items of each component. The shape parameter $\beta_{i}$ and the scale parameter $\theta_{i}$ are obtained from the study of Laggoune et al (2009), where failure data were sampled to estimate the two parameters. The cost parameters related to inspection and hidden failure are introduced for illustration purpose, including the individual inspection $\operatorname{cost} c_{I}^{i}$ and the individual downtime cost $c_{d}^{i}$.

Based on the discussions in Section 3.2.1, we obtain the optimal inspection interval $\tau_{i}^{*}$ and the associated long-run cost rate $G_{i}^{*}\left(\tau_{i}\right)$ for each component, as shown in Table 4. For the purpose of illustration, we also plot the variation of $G_{i}\left(\tau_{i}\right)$ with respect to $\tau_{i}$. Four components are selected for illustration: tightness (C275), stub bearing (C230), carrying bearing (C419) and stub bearing (C401). Fig 7 shows how $G_{i}\left(\tau_{i}\right)$ varies with $\tau_{i}$ of the four components.

Table 4 Optimal inspection policy for individual component

\begin{tabular}{cccc}
\hline Component & code & $\begin{array}{c}\text { Optimal individual } \\
\text { inspection interval } \\
\tau_{i}^{*}(\text { day })\end{array}$ & $\begin{array}{c}\text { Optimal individual } \\
\text { long-run cost } \\
\text { rate } G_{i}^{*}\left(\tau_{i}\right)(€)\end{array}$ \\
\hline Sheathing & C286 & 58 & 37 \\
Sheathing & C285 & 17 & 65 \\
Tightness & C275 & 15 & 275 \\
Stub bearing & C230 & 36 & 159 \\
Tightness ring & C460 & 19 & 36 \\
Carrying bearing & C419 & 18 & 175 \\
Stub bearing & C401 & 48 & 269 \\
Labyrinth support & C780 & 54 & 390 \\
\hline
\end{tabular}




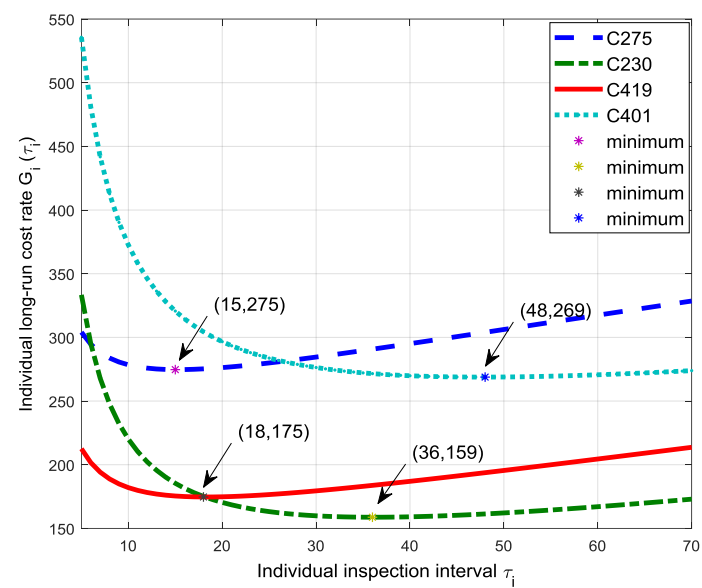

Fig 7 Plot of individual long-run cost rate

The common downtime cost is given as $C_{I}=€ 2500$. According to Eq (7), the variation of long-run cost rate $G(\tau)$ with respect to the base interval $\tau$ is plotted in Fig 8. From Fig 8, the minimum long-run cost rate of $€ 1517$, obtained at the optimal base interval $\tau^{*}=29$ days. After obtaining the optimal base interval $\tau^{*}$, we can determine the integral multiple $k_{i}$ for each component. The result is shown in Table 5. By comparing the results of Table 5 with the optimal individual inspection interval, it is observed that the inspection period of stub bearing (C230) is shifted in advance, the inspection period of sheathing (C286) remains unchanged, and the inspection period of other components are postponed. Proposition 2 implies that the upper bound of the base interval policy is at most $\sup \left\{\left(\mu_{i}+\tau_{i}^{*}\right) / \mu_{i}: i=1,2, . ., 8\right\}=1.201$ times of the lower bound. In this section, the lower bound can be computed as $L B=1501$. The long-run cost rate obtained by base interval approach is actually 1.011 times of the lower bound. 


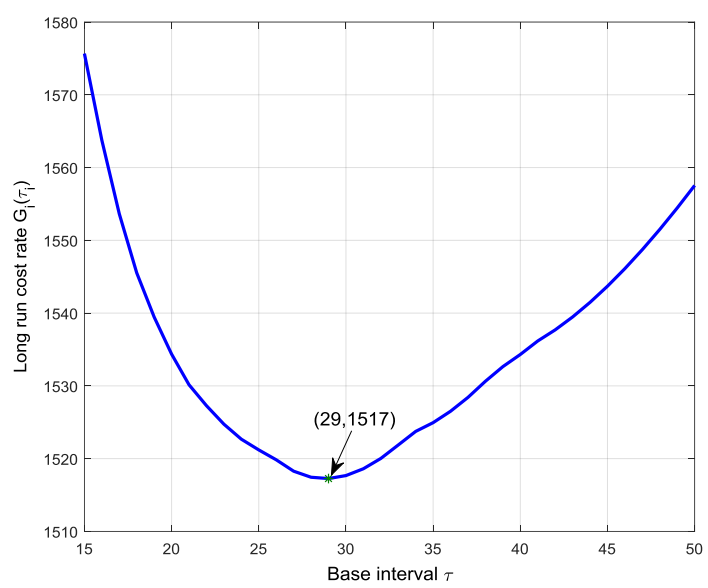

Fig 8 Variation of $G(\tau)$ with respect to base interval $\tau$

Table 5 Optimal inspection interval for base interval policy

\begin{tabular}{cccc}
\hline Component & code & $\begin{array}{c}k_{i} \text { associated with } \\
\text { optimal base interval }\end{array}$ & $\begin{array}{c}\text { Optimal inspection } \\
\text { interval } k_{i} \tau \text { (days) }\end{array}$ \\
\hline Sheathing & C286 & 2 & 58 \\
Sheathing & C285 & 1 & 29 \\
Tightness & C275 & 1 & 29 \\
Stub bearing & C230 & 1 & 29 \\
Tightness ring & C460 & 1 & 29 \\
Carrying bearing & C419 & 1 & 29 \\
Stub bearing & C401 & 2 & 58 \\
Labyrinth support & C780 & 2 & 58 \\
\hline
\end{tabular}

Furthermore, in order to investigate how the components are grouped with various base interval $\tau$, we plot $k_{i}$ with respect to $\tau$ in Fig 9. When $\tau$ increases from 15 to 50 days, $k_{i}$ of the components show a non-increasing trend. When $\tau$ is no less than 41 days, $k_{i}$ reduces to 1 for $i=1,2, \ldots, 8$, which implies that all the components are inspected at the same time. Note that we only plot $k_{i}$ of four components (C286, C230, C401 and C780). $k_{i}$ of the other four components are not shown in Fig 6, as they always remain 1 when $\tau$ varies within the range of $[15,50]$. 


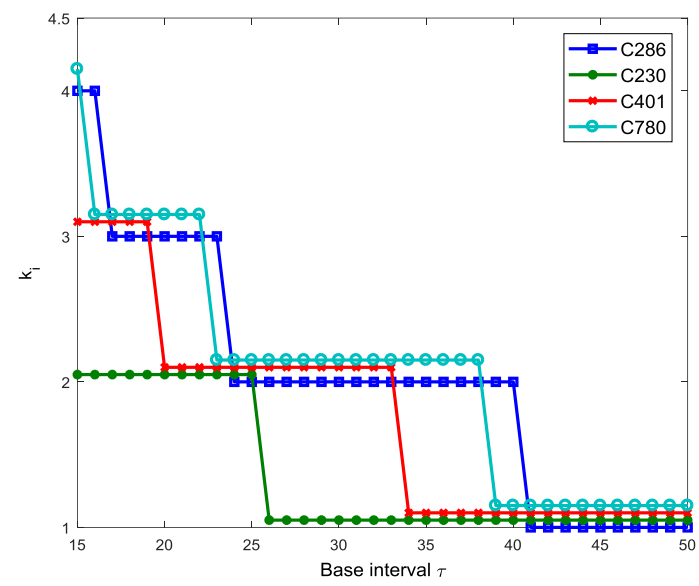

Fig 9 Plot of $k_{i}$ with respect to base interval $\tau$

Fig 10 shows how the optimal base interval $\tau^{*}$ and the optimal long-run cost rate $G^{*}(\tau)$ vary with the common downtime cost $C_{I}$. When $C_{I}$ increases from $€ 500$ to $€ 4500, G^{*}(\tau)$ increase monotonically from $€ 1434$ to $€ 1582$. In addition, the optimal base interval $\tau^{*}$ shows an increasing trend with $C_{I}$, changing from 9 to 26 days. The result indicates that common downtime cost has a significant influence on the optimal inspection policy. It is therefore suggested that maintenance engineers or managers should take into account the common cost (interactions among components) when making maintenance decisions for a multi-component system.

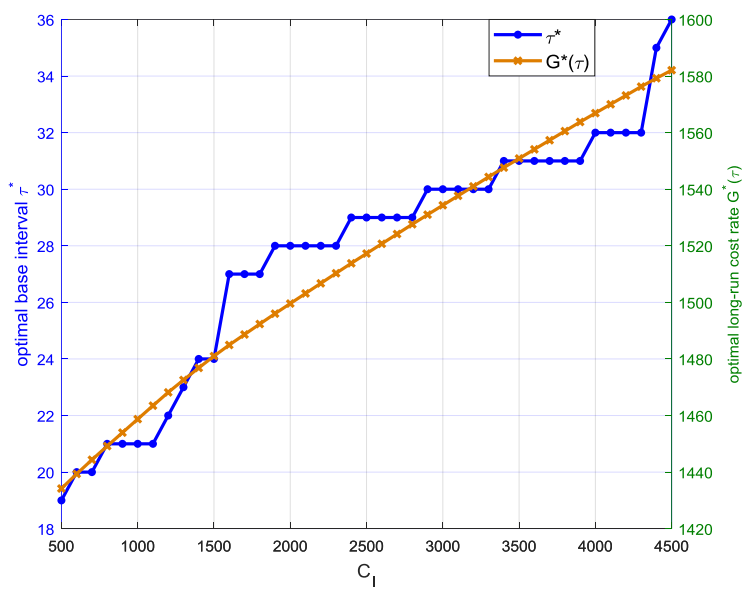

Fig 10 Sensitivity analysis on $C_{I}$ 


\section{Conclusions}

This paper develops a maintenance policy for multi-component systems subject to hidden failures. Failure of a component can only be detected at inspection and a failed component is replaced once the failure is revealed. Different from previous works where an optimal inspection interval is applied for the whole system, we obtain the optimal inspection interval for each component. Concerned with the difficulty of the optimization problem, a heuristic method named base interval approach is adopted to reduce the computational complexity. Upper bound of the policy is analyzed, followed by two numerical examples. It is illustrated that that the base interval policy can approximate the optimal policy within a small factor. The result of a numerical example shows that our approach outperforms the individual inspection policy and the common inspection policy.

The proposed maintenance strategy can be applied in various complex systems such as power generators and processing facilities, especially for systems with a high common downtime cost. One advantage of the proposed maintenance policy lies in its feasibility and applicability in industry. Maintenance crews only need to inspect the components at fixed intervals and replace the failed components, while no other tricky implementations are required.

Future research can be conducted by extending the present inspection-replacement policy into a more general maintenance context. For example, consider a multi-component reparable system where various maintenance actions such as minimal repair and imperfect maintenance are allowed. In addition, if the components of the system follow a continuously degrading process and the degrading state can be observed by inspection devices, one can integrate the concept of base interval approach into condition-based maintenance and develop a more efficient maintenance policy.

\section{Acknowledgement}

The work described in this paper was supported by University Grants Council of Hong Kong under a theme-based project grant (T32-101/15-R) and a GRF (CityU 11203815), and also by National Natural Science Foundation of China under a Key Project (71532008). 


\section{References}

Ahmadi, A., \& Kumar, U. (2011). Cost based risk analysis to identify inspection and restoration intervals of hidden failures subject to aging. IEEE Transactions on Reliability, 60(1), 197-209.

Atkins, D., \& Iyogun, P. (1987). A lower bound on a class of coordinated inventory/production problems. Operations Research Letters, 6(2), 63-67.

Badia, F. G., Berrade, M. D., \& Campos, C. A. (2001). Optimization of inspection intervals based on cost. Journal of Applied Probability, 38(4), 872-881.

Camci, F. (2009). System maintenance scheduling with prognostics information using genetic algorithm. IEEE Transactions on Reliability, 58(3), 539-552.

Cheng, Y., Chen, X., Ren, J., Xuan, X., \& Li, X. (2013). Study on hidden failure of relay protection in power system. IEEE Annual International Conference on Cyber Technology in Automation Control and Intelligent Systems, Nanjing, China, 434-439.

Gao, Y., Feng, Y., Zhang, Z., \& Tan, J. (2015). An optimal dynamic interval preventive maintenance scheduling for series systems. Reliability Engineering \& System Safety, 142, 19-30.

Gustavsson, E., Patriksson, M., Strömberg, A. B., Wojciechowski, A., \& Önnheim, M. (2014). Preventive maintenance scheduling of multi-component systems with interval costs. Computers \& industrial engineering, 76, 390-400.

He, K., Maillart, L. M., \& Prokopyev, O. A. (2015). Scheduling Preventive Maintenance as a Function of an Imperfect Inspection Interval. IEEE Transactions on Reliability, 64(3), 983-997.

Hopp, W. J., \& Kuo, Y. L. (1998). Heuristics for multi-component joint replacement: Applications to aircraft engine maintenance. Naval Research Logistics, 45(5), 435-458.

Huynh, K. T., Barros, A., \& Berenguer, C. (2015). Multi-Level Decision-Making for The Predictive Maintenance of-Out-of-: F Deteriorating Systems. IEEE Transactions on Reliability, 64(1), 94-117. 
Jackson, P., Maxwell, W., \& Muckstadt, J. (1985). The joint replenishment problem with a powers-of-two restriction. IIE Transactions, 17(1), 25-32.

Laggoune, R., Chateauneuf, A., \& Aissani, D. (2009). Opportunistic policy for optimal preventive maintenance of a multi-component system in continuous operating units. Computers \& Chemical Engineering, 33(9), 1499-1510.

Lam, J. Y. J., \& Banjevic, D. (2015). A myopic policy for optimal inspection scheduling for condition based maintenance. Reliability Engineering \& System Safety, 144, 1-11.

Levi, R., Magnanti, T., Muckstadt, J., Segev, D., \& Zarybnisky, E. (2014). Maintenance scheduling for modular systems: Modeling and algorithms. Naval Research Logistics (NRL), 61(6), 472-488.

Liu, B., Wu, J., \& Xie, M. (2015). Cost analysis for multi-component system with failure interaction under renewing free-replacement warranty. European Journal of Operational Research, 243(3), 874-882.

Liu, B., Wu, S., Xie, M., \& Kuo, W. (2017). A condition-based maintenance policy for degrading systems with age-and state-dependent operating cost. European Journal of Operational Research, 263(3), 879-887.

Liu, B., Xie, M., Xu, Z., \& Kuo, W. (2016). An imperfect maintenance policy for missionoriented systems subject to degradation and external shocks. Computers \& Industrial Engineering, 102, 21-32.

Liu, B., Xu, Z., Xie, M., \& Kuo, W. (2014). A value-based preventive maintenance policy for multi-component system with continuously degrading components. Reliability Engineering \& System Safety, 132, 83-89.

Nicolai, R. P., \& Dekker, R. (2008). Optimal maintenance of multi-component systems: a review (pp. 263-286). Springer London.

Pandey, M., Zuo, M. J., \& Moghaddass, R. (2015). Selective maintenance scheduling over a finite planning horizon. Proceedings of the Institution of Mechanical Engineers, Part O: Journal of Risk and Reliability, 230(2), 162-177. 
Peng, H., Feng, Q., \& Coit, D. W. (2010). Reliability and maintenance modeling for systems subject to multiple dependent competing failure processes. IIE Transactions, 43(1), 12-22.

Phan, D. T., \& Zhu, Y. (2015). Multi-stage optimization for periodic inspection planning of geodistributed infrastructure systems. European Journal of Operational Research, 245(3), 797-804.

Taghipour, S., \& Banjevic, D. (2011). Periodic inspection optimization models for a repairable system subject to hidden failures. IEEE Transactions on Reliability, 60(1), 275-285.

Tian, Z., \& Liao, H. (2011). Condition based maintenance optimization for multi-component systems using proportional hazards model. Reliability Engineering \& System Safety, 96(5), 581589.

Vu, H. C., Do, P., Barros, A., \& Berenguer, C. (2014). Maintenance grouping strategy for multicomponent systems with dynamic contexts. Reliability Engineering \& System Safety, 132, 233249.

Wang, H. (2002). A survey of maintenance policies of deteriorating systems. European Journal of Operational Research, 139(3), 469-489.

Wang, H. K., Huang, H. Z., Li, Y. F., \& Yang, Y. J. (2016). Condition-Based Maintenance With Scheduling Threshold and Maintenance Threshold. IEEE Transactions on Reliability, 65(2), 513-524.

Wang, Y., \& Pham, H. (2011). A multi-objective optimization of imperfect preventive maintenance policy for dependent competing risk systems with hidden failure. IEEE Transactions on Reliability, 60(4), 770-781.

Xiao, L., Song, S., Chen, X., \& Coit, D. W. (2016). Joint optimization of production scheduling and machine group preventive maintenance. Reliability Engineering \& System Safety, 146, 68-78.

Xiao, X., \& Ye, Z. (2016). Optimal design for destructive degradation tests with random initial degradation values using the wiener process. IEEE Transactions on Reliability, 65(3), 1327-1342. 
Yang, F., Meliopoulos, A. S., Cokkinides, G. J., \& Binh Dam, Q. (2006). Effects of protection system hidden failures on bulk power system reliability. Proceedings of the 38th Annual North American Power Symposium, 517-523.

Ye, Z., Revie, M., \& Walls, L. (2014). A Load Sharing System Reliability Model With Managed Component Degradation. IEEE Transactions on Reliability, 63(3), 721-730.

Ye, Z. S., \& Xie, M. (2015). Stochastic modelling and analysis of degradation for highly reliable products. Applied Stochastic Models in Business and Industry, 31(1), 16-32.

Zhang, M., Gaudoin, O., \& Xie, M. (2015). Degradation-based maintenance decision using stochastic filtering for systems under imperfect maintenance. European Journal of Operational Research, 245(2), 531-541.

Zhu, W., Fouladirad, M., \& Berenguer, C. (2016). A multi-level maintenance policy for a multicomponent and multifailure mode system with two independent failure modes. Reliability Engineering \& System Safety, 153, 50-63.

\section{Appendix}

1. Proof of Proposition 1.

$G_{i}\left(\tau_{i}\right)$ can be rewritten as

$$
G_{i}\left(\tau_{i}\right)=c_{d}^{i}+\frac{U\left(\tau_{i}\right)}{\tau_{i}}
$$

where $U\left(\tau_{i}\right)=\left(-c_{d}^{i} \mu_{i}+c_{r}^{i}+c_{I}^{i} \sum_{j=0}^{\infty} R_{i}\left(j \tau_{i}\right)\right) / \sum_{j=0}^{\infty} R_{i}\left(j \tau_{i}\right)$.

After some simplifications, $U\left(\tau_{i}\right)$ is expressed as $U\left(\tau_{i}\right)=c_{I}^{i}-\left(c_{d}^{i} \mu_{i}-c_{r}^{i}\right) / \sum_{j=0}^{\infty} R_{i}\left(j \tau_{i}\right)$. Obviously, $\sum_{j=0}^{\infty} R_{i}\left(j \tau_{i}\right)$ is a decreasing function of $\tau_{i}$ and $\lim _{\tau_{i} \rightarrow \infty} \sum_{j=0}^{\infty} R_{i}\left(j \tau_{i}\right)=1$. Suppose that $c_{d}^{i} \mu_{i}-c_{r}^{i}<c_{I}^{i}$, then we have $G_{i}\left(\tau_{i}\right)>c_{d}^{i}, \forall \tau_{i} \in(0, \infty)$. Moreover, $\lim _{\tau_{i} \rightarrow \infty} \sum_{j=0}^{\infty} R_{i}\left(j \tau_{i}\right)=1$ implies that $\lim _{\tau_{i} \rightarrow \infty} G_{i}\left(\tau_{i}\right)=c_{d}^{i}$, leading to the conclusion that $\tau_{i}^{*}=\infty$. 
If the condition $c_{d}^{i} \mu_{i}-c_{r}^{i}>c_{I}^{i}$ holds, we can conclude that $U\left(\tau_{i}\right)$ is decreasing with $\tau_{i}$. Also, we have

$$
\begin{aligned}
& \lim _{\tau_{i} \rightarrow 0} U\left(\tau_{i}\right)=c_{I}^{i}>0 \\
& \lim _{\tau_{i} \rightarrow \infty} U\left(\tau_{i}\right)=c_{I}^{i}-\left(c_{d}^{i} \mu_{i}-c_{r}^{i}\right)<0
\end{aligned}
$$

The monotonicity of $U\left(\tau_{i}\right)$ and the above limit property imply that there exists a $\tau_{i}^{0}$ such that

$$
\begin{aligned}
& U\left(\tau_{i}\right)>0, \forall \tau_{i} \in\left(0, \tau_{i}^{0}\right) \\
& U\left(\tau_{i}\right)<0, \forall \tau_{i} \in\left(\tau_{i}^{0}, \infty\right)
\end{aligned}
$$

Hence, $G_{i}\left(\tau_{i}\right)$ has the following properties:

$$
\begin{gathered}
G_{i}\left(\tau_{i}^{0}\right)=c_{d}^{i} \\
\lim _{\tau_{i} \rightarrow \infty} G_{i}\left(\tau_{i}\right)=c_{d}^{i} \\
G_{i}\left(\tau_{i}\right)>c_{d}^{i}, \forall \tau_{i} \in\left(0, \tau_{i}^{0}\right) \\
G_{i}\left(\tau_{i}\right)<c_{d}^{i}, \forall \tau_{i} \in\left(\tau_{i}^{0}, \infty\right)
\end{gathered}
$$

The above properties imply that there exits an optimal $\tau_{i}^{*} \in\left(\tau_{i}^{0}, \infty\right)$ such that $G_{i}\left(\tau_{i}\right)$ reaches its minimum. The optimal inspection interval $\tau_{i}^{*}$ can be obtained by setting the derivative to zero, i.e., $\tau_{i}^{*} \in\left\{\tau_{i}: d G_{i}\left(\tau_{i}\right) / d \tau_{i}=0\right\}$.

\section{Proof of lemma 1.}

For notational simplicity, define $Q\left(\tau_{i}\right)=\sum_{j=0}^{\infty} R_{i}\left(j \tau_{i}\right)$. By definition, we have

$$
\frac{\mu_{i}}{\tau_{i}} \leq\left\lceil\frac{\mu_{i}}{\tau_{i}}\right\rceil<\frac{\mu_{i}}{\tau_{i}}+1
$$

Taking expectation of the above expression leads to

$$
\frac{\mu_{i}}{\tau_{i}} \leq Q\left(\tau_{i}\right)=E\left[\left[\frac{\mu_{i}}{\tau_{i}}\right]\right]<\frac{\mu_{i}}{\tau_{i}}+1
$$

Thus, we have 


$$
\mu_{i} \leq \tau_{i} Q\left(\tau_{i}\right)<\mu_{i}+\tau_{i}
$$

The individual optimal inspection interval $\tau_{i}^{*}$ is limited within the range

$$
\left(k_{i}-1\right) \tau<\tau_{i}^{*} \leq k_{i} \tau
$$

Then, we can obtain

$$
G_{i}\left(k_{i} \tau\right)=\frac{-c_{d}^{i} \mu_{i}+c_{r}^{i}+c_{I}^{i} Q\left(k_{i} \tau\right)}{k_{i} \tau S\left(k_{i} \tau\right)} \leq \frac{-c_{d}^{i} \mu_{i}+c_{r}^{i}+c_{I}^{i} Q\left(k_{i} \tau\right)}{\mu_{i}} \leq \frac{-c_{d}^{i} \mu_{i}+c_{r}^{i}+c_{I}^{i} Q\left(\tau_{i}^{*}\right)}{\mu_{i}}
$$

The first inequality is due to the expression that $\mu_{i} \leq \tau_{i} Q\left(\tau_{i}\right)$; the second inequality is due to the fact that $\tau_{i}^{*} \leq k_{i} \tau$ and that $Q\left(\tau_{i}\right)$ is a non-increasing function. The follows the inequality

$$
G_{i}\left(\tau_{i}^{*}\right)=\frac{-c_{d}^{i} \mu_{i}+c_{r}^{i}+c_{I}^{i} Q\left(\tau_{i}^{*}\right)}{\tau_{i}^{*} Q\left(\tau_{i}^{*}\right)}>\frac{-c_{d}^{i} \mu_{i}+c_{r}^{i}+c_{I}^{i} Q\left(\tau_{i}^{*}\right)}{\mu_{i}+\tau_{i}^{*}}
$$

Therefore, we have

$$
\frac{G_{i}\left(k_{i} \tau\right)}{G_{i}\left(\tau_{i}^{*}\right)}<\frac{\mu_{i}+\tau_{i}^{*}}{\mu_{i}}
$$

which completes the proof. $\square$

\section{Proof of Proposition 2.}

Let the inspection interval of component $i$ is $\tau$ for $i=1,2, \ldots, \kappa$ and $k_{i} \tau$ for $i=\kappa+1, \kappa+2, \ldots, n$. As shown in lemma 1,

$$
\frac{G_{i}\left(k_{i} \tau\right)}{G_{i}\left(\tau_{i}^{*}\right)}<\frac{\mu_{i}+\tau_{i}^{*}}{\mu_{i}}
$$

the upper bound is 


$$
\begin{aligned}
& U B=\frac{C_{I}}{\tau}+\sum_{i=1}^{\kappa} G_{i}(\tau)+\sum_{i=\kappa+1}^{n} G_{i}\left(k_{i} \tau\right) \\
& <\frac{C_{I}}{\tau}+\sum_{i=1}^{\kappa} G_{i}(\tau)+\sum_{i=\kappa+1}^{n} \frac{\mu_{i}+\tau_{i}^{*}}{\mu_{i}} G_{i}\left(\tau_{i}^{*}\right) \\
& <\frac{C_{I}}{\tau}+\sum_{i=1}^{\kappa} G_{i}(\tau)+\sup \left\{\frac{\mu_{i}+\tau_{i}^{*}}{\mu_{i}}: i=1,2, \ldots, n\right\} \sum_{i=\kappa+1}^{n} G_{i}\left(\tau_{i}^{*}\right) \\
& <\sup \left\{\frac{\mu_{i}+\tau_{i}^{*}}{\mu_{i}}: i=1,2, \ldots, n\right\}\left(\frac{C_{I}}{\tau}+\sum_{i=1}^{\kappa} G_{i}(\tau)+\sum_{i=\kappa+1}^{n} G_{i}\left(\tau_{i}^{*}\right)\right) \\
& =\sup \left\{\frac{\mu_{i}+\tau_{i}^{*}}{\mu_{i}}: i=1,2, \ldots, n\right\} L B
\end{aligned}
$$

which concludes the proof. $\square$

Bin Liu received his B.S. degree from the Department of Automation, Zhejiang University, Zhejiang, China, in 2013.

$\mathrm{He}$ is currently completing the Ph.D. degree in Department of Systems Engineering \& Engineering Management, City University of Hong Kong, Hong Kong. His research interests include reliability and maintenance modeling, importance measures with application to complex systems and data analysis.

Ruey-Huei Yeh received his B.S. degree in industrial engineering and engineering management from the National Tsing Hua University, Taiwan, in 1984, and M.S. \& Ph.D. degrees in industrial and operations engineering from the University of Michigan, Ann Arbor, U.S.A. in 1989 and 1993, respectively.

$\mathrm{He}$ is a Distinguished Professor at the Industrial Management Department, National Taiwan University of Science and Technology (Taiwan Tech.), Taiwan. He joined the National Taiwan University of Science and Technology as an Associate Professor and became a full professor in 1999. He has received several teaching and research awards from the University, and awarded as a Distinguished Professor in 2012. His research interests cover reliability analysis, decision analysis, quality control, and warranty policies. 
Min Xie (M'91-SM'94-F'06) received the Ph.D. degree in quality technology from Linkoping University, Linkoping, Sweden, in 1987.

Currently, he is a Chair Professor at City University of Hong Kong. He has authored or coauthored numerous refereed journal papers, and some books on quality and reliability engineering, including Software Reliability Modeling (World Scientific), Weibull Models (Wiley), Computing Systems Reliability (Kluwer), and Advanced QFD Applications (ASQ Quality Press).

Prof. Xie was awarded the prestigious LKY Research Fellowship in 1991. He is a Department Editor of IIE Transactions, Associate Editor of Reliability Engineering and System Safety, and is on the editorial board of a number other international journals.

Way Kuo (M'83-SM'86-F'92) received the B.S. degree in nuclear engineering from the National Tsing Hua University, Hsinchu, Taiwan, R.O.C., in 1972, and the Ph.D. degree in industrial engineering from Kansas State University, Manhattan, in 1980.

$\mathrm{He}$ is President of City University of Hong Kong. Before joining CityU, he was Dean of Engineering at the University of Tennessee, Knoxville. Previously, he was with Texas A\&M University, Iowa State University, and Bell Labs.

Prof. Kuo is a member of US National Academy of Engineering and Academia Sinica in Taiwan, and a foreign member of Chinese Academy of Engineering. He has co-authored several books and served as Editor-in-Chief of IEEE Transactions on Reliability. 